\section{La depresión como un diagnóstico complejo. Implicancias para el desarrollo de recomendaciones clínicas}

\author{
ALBERTO BOTTO, JULIA ACUÑA, JUAN PABLO JIMÉNEZ
}

Depression has a high impact on mental health. However its diagnosis is a challenge even for specialists. This problem derives from a failure in an adequate description and differentiation of the disease. This inadequate conceptualization generates these difficulties. Our thesis is that depression should be understood as a complex phenomenon that can be analyzed from multiple perspectives, from genes to behavior, including personality and interaction with the sociocultural environment. The aim of this paper is to review the psychopathological construct of depression from a multidimensional point of view, considering clinical, sociocultural, characterological and pathogenic variables. Finally we provide a proposal for an adequate diagnostic approach.

(Rev Med Chile 2014; 142: 1297-1305)

Key words: Depression; diagnosis; Psychopathology.

\author{
Departamento de Psiquiatría y Salud \\ Mental Oriente, Facultad de Medicina, \\ Universidad de Chile. \\ Financiado por: 1) Proyecto Fonis \\ SA09120014 "Impacto de un modelo de \\ capacitación en trastornos depresivos, \\ integrado con la práctica reflexiva, \\ sobre las habilidades diagnósticas, \\ terapéuticas y resolutivas del médico \\ general en atención primaria de salud"; \\ 2) Iniciativa Científica Milenio del \\ Ministerio de Economía, Fomento y \\ Turismo NS 100018. Núcleo Milenio \\ "Intervención Psicológica y Cambio en \\ Depresión". \\ Nota: Las organizaciones que \\ proporcionaron ayuda financiera \\ no tuvieron influencia alguna en la \\ preparación, revisión o aprobación del \\ manuscrito. \\ Recibido el 5 de diciembre de 2013, \\ aceptado el 27 de agosto de 2014. \\ Correspondencia a: \\ Alberto Botto \\ Departamento de Psiquiatría y Salud \\ Mental Oriente. \\ Av. Salvador 486, Providencia, Santiago \\ de Chile. \\ abottov@gmail.com
}

L a depresión es una enfermedad recurrente, frecuentemente crónica y que requiere un tratamiento a largo plazo ${ }^{1}$. Su prevalencia en la población general se encuentra entre 8 y $12 \%^{2}$. Se estima que para el año 2030 ocupará el primer lugar en la medición de carga de enfermedad según los años de vida ajustados por discapacidad $^{3}$. A pesar de esto, su diagnóstico aún continúa planteando múltiples desafíos tanto conceptuales como clínicos. Con el objeto de resolver los problemas de validez y confiabilidad de los criterios diagnósticos, en la actualidad se ha impuesto un modelo ateórico, (representado por el Diagnostic and Statistic Manual of Mental Disorders, DSM y la Clasificación Internacional de Enfermedades, CIE) cuya utilidad ha sido cuestionada, no encontrándose hasta el momento una solución definitiva ${ }^{4}$. Ante esta realidad clínica subyace un problema grave y complejo. La evidencia muestra que para los propios psiquiatras existen importantes dificultades al momento de plantear un diagnóstico que resulte adecuado para el cuidado de los pacientes depresivos ${ }^{5}$ y no es de extrañar que el médico general tenga al menos las mismas dificultades. Así, se ha visto que en atención primaria de salud (APS), la depresión suele ser sobrediagnosticada, subdiagnosticada y tratada de manera insuficiente ${ }^{6-8}$.

El objetivo de este trabajo es revisar el constructo de la depresión desde el punto de vista del diagnóstico psiquiátrico, considerando aspectos clínicos ("espectro afectivo"), socioculturales, etiopatogénicos y caracterológicos. La tesis que guía esta revisión es que la depresión debe comprenderse según una concepción de la relación mente-cerebro, entendida como un sistema complejo y que, por lo tanto, debe ser analizada desde múltiples perspectivas9. Para eso, plantearemos un modelo integrado que facilite al médico el buen desarrollo de su juicio clínico mientras trabaja con 
sus pacientes. Finalmente, entregaremos algunas recomendaciones respecto al abordaje clínico del diagnóstico de la depresión.

\section{El espectro afectivo}

El término "depresión” surgió a mediados del siglo XIX, para referirse a una alteración primaria de las emociones cuyos rasgos más sobresalientes reflejaban menoscabo, inhibición y deterioro funcional $^{10,11}$. En 1980 se publica el DSM-III, donde por primera vez se incorpora el diagnóstico de "depresión mayor”. Sin embargo, en la serie DSM, la depresión es entendida como una entidad homogénea donde, dada su orientación eminentemente "sintomática", muchos aspectos psicopatológicos dejan de ser considerados ${ }^{12}$. Lo anterior contrasta con la evidencia actual que apoya la existencia de diversas formas clínicas cuya identificación es relevante como, por ejemplo, la depresión melancólica, atípica y psicótica; la depresión bipolar; subtipos como la depresión estacional o con predominio de síntomas somáticos, crisis de pánico o síntomas obsesivos; la depresión que acompaña a enfermedades físicas y, por último, la pseudodemencia depresiva ${ }^{13}$. Así, un cuidadoso análisis psicopatológico resulta fundamental para la determinación de "endofenotipos de respuesta" a través de los cuales es posible predecir la evolución individual frente los tratamientos ${ }^{14}$.

En la actualidad, los sistemas clasificatorios se han mostrado insuficientes para dar cuenta del fenómeno clínico de la depresión. Así, el concepto de "depresión mayor" parece no ser una entidad suficientemente válida y confiable por lo que, en consecuencia, no otorga un campo sólido sobre el cual se puedan elaborar teorías etiopatogénicas o discutir acerca de la respuesta diferencial de los tratamientos ${ }^{15}$. Históricamente, el análisis del constructo "depresión" ha considerado dos modelos comprensivos: el unitario y el binario. El modelo unitario plantea que la depresión es el resultado de una vía final común psicobiológica, independientemente de sus causas o desencadenantes ${ }^{16}$. Según éste, la depresión es una entidad única que varía sólo en función de su gravedad. El modelo binario, por el contrario, considera dos subtipos de depresiones (cada una con mecanismos etiopatogénicos diferentes): las endógenas/ psicóticas y las neuróticas/reactivas. Sin embargo, existe un tercer modelo, el jerárquico ${ }^{17}$, según el cual la alteración psicomotora es el rasgo fundamental de la depresión melancólica ${ }^{18}$. La depresión no melancólica se presenta en el contexto de múltiples cuadros donde se incluyen los trastornos de ansiedad y los trastornos de personalidad. La variedad de síntomas asociados con las depresiones atípicas constituye un argumento para postular un espectro, lo que supone que los estilos de personalidad contribuyen a la configuración del cuadro clínico -tanto de los síntomas como de las respuestas de afrontamiento- y, por lo tanto, permiten una mejor identificación fenotípica ${ }^{19}$. De esta manera se ha planteado que el episodio depresivo mayor incluye cuatro diferentes tipos de depresión dentro de un espectro que va desde el polo más crónico y leve hasta el polo más episódico y severo ${ }^{20}$.

Desde mediados de la década de los sesenta las evidencias avalan la existencia de una continuidad de las manifestaciones anímicas -el "espectro afectivo"- que abarca desde el polo más "caracterológico", representado por la distimia, hasta el polo más "biológico" representado por la enfermedad bipolar de tipo I, pasando en orden secuencial por la depresión mayor unipolar, la depresión crónica, la depresión atípica, la depresión psicótica, la depresión recurrente, los trastornos del espectro bipolar y la enfermedad bipolar de tipo $\mathrm{II}^{21,22,23,24}$. Según esta hipótesis, los cuadros afectivos se entienden mejor desde una perspectiva dimensional y longitudinal. Así, se ha sugerido que no es posible plantear una distinción categórica entre cuadros bipolares y unipolares; por el contrario, se piensa que las fluctuaciones anímicas son fenómenos compartidos en distinto grado por ambas entidades. Las implicancias de esta aproximación no son sólo teóricas, ya que la sola presencia de manifestaciones esporádicas sugerentes de hipomanía/ manía en pacientes con cuadros pseudounipolares apuntan hacia un diagnóstico, un pronóstico y un tratamiento específicos. Por otra parte, el elevado porcentaje de comorbilidad con trastornos de ansiedad $^{25}$, más que corroborar los respectivos diagnósticos, cuestiona la legitimidad de su separación conceptual, haciendo su evaluación clínica aún más dificultosa.

En relación con lo anterior podemos plantear que, desde el punto de vista clínico, la depresión es una entidad compleja que debe ser comprendida dimensionalmente en el contexto del espectro de los trastornos afectivos. A través de las manifesta- 
ciones psicopatológicas particulares es necesario diferenciar grupos clínicos o endofenotipos que condicionarán la indicación del tratamiento.

\section{La construcción socio-cultural de la depresión}

Los sentimientos depresivos de tristeza pueden ser experimentados por todas las personas $\mathrm{y}$ forman parte de las manifestaciones afectivas normales frente al duelo y la pérdida. Sin embargo, la manera en que la depresión es entendida, interpretada, discutida, comunicada y tratada, varía entre una cultura y otra. Por ejemplo, en algunas lenguas del sudeste asiático no existe un equivalente a la palabra "deprimido", mientras que en países como Nigeria se utiliza sólo una palabra para designar la depresión, la ansiedad y la rabia ${ }^{26}$.

En las sociedades occidentales es más frecuente concebir la depresión como una "enfermedad" con componentes hereditarios y biológicos, mientras que una visión "situacional" que comprende los síntomas en el contexto de un estrés psicosocial y de dificultades interpersonales suele asociarse con sociedades tradicionales y comunidades minoritarias. Es así como quienes interpretan los problemas como "reacciones emocionales" frente a las adversidades del entorno -al contrario de una interpretación "patológica"- son menos propensos a buscar ayuda profesional en el ámbito de la salud mental ${ }^{27}$. Otros, en cambio, se sentirán más cómodos cuando se establece una relación directiva y jerárquica, mucho más cercana al modelo médico clásico. En estudios realizados en comunidades chinas, por ejemplo, la experiencia de depresión es más "física" que "psicológica"; rara vez los pacientes reportan sentimientos de tristeza o desánimo; en cambio, presentan molestias como dolor, somnolencia, fatiga y aburrimiento, evidenciando un patrón claramente somatomor$\mathrm{fo}^{28}$. Esto se explica porque en estas comunidades la expresión de sentimientos, especialmente de afectos negativos, se considera algo culturalmente reprobable.

Las diferencias en el modo en que varía la frecuencia de síntomas somáticos de la depresión puede deberse, en parte, a características de los médicos y los sistemas de atención en salud así como también de variables culturales entre los pacientes $^{29}$. Estas observaciones apuntan a que aunque existen elementos universales en la psicopatología de la depresión, la manera en que ésta se expresa se encuentra modelada por la cultura.

\section{Modelos etiopatogénicos de la depresión}

La psicopatología de la depresión no sólo varía entre los distintos pacientes sino también entre los distintos episodios dentro de la vida de un mismo paciente. Este pleomorfismo clínico constituye un importante desafío en el estudio de su etiopatogenia ${ }^{30}$.

En el origen de la depresión interactúan factores genéticos y ambientales. Su heredabilidad se ha estimado en estudios de gemelos entre $31-42 \%$ con una contribución ambiental de $58-67 \%{ }^{31}$. Los modelos biológicos de la depresión incluyen una larga serie de genes ${ }^{32}$, siendo uno de los más estudiados el que codifica el transportador de serotonina ${ }^{33}$. Además, se ha visto que los subtipos de depresión se relacionan con patrones específicos de alteración ${ }^{34}$. Las experiencias traumáticas infantiles pueden contribuir con la aparición de depresión en la adultez ${ }^{35}$, especialmente en aquellos individuos más vulnerables ${ }^{36}$. Incluso se ha postulado la existencia de subtipos neurobiológicos de depresión dependiendo de la presencia o ausencia de eventos tempranos adversos ${ }^{37}$. Recientemente se ha sugerido que diversos procesos inflamatorios jugarían un rol en la etiopatogenia de la depresión no sólo como precipitantes sino también como factores mantenedores de la sintomatología ${ }^{38}$.

Sigmund Freud distinguió por primera vez los dinamismos psicológicos que subyacen a las reacciones afectivas de duelo y los síntomas de la depresión melancólica ${ }^{39}$, postulando que lo característico de la melancolía es una pérdida de la autoestima manifestada clínicamente por la presencia de autorreproches. Posteriormente, el modelo cognitivo-conductual de la depresión postuló la existencia de una serie de cogniciones (concientes) erradas o distorsionadas respecto a la manera de interpretar las experiencias ${ }^{40}$.

Aunque las hipótesis evolucionistas sobre el origen de la depresión se han postulado desde hace algún tiempo, no han dejado de ser controversiales $^{41}$. En la depresión, entendida como un fenómeno adaptativo, el desánimo y los síntomas asociados contribuyen con el manejo de situaciones inapropiadas o potencialmente dañinas, por 
ejemplo, comunicando una necesidad de ayuda o actuando como señal de sumisión en conflictos sociales en torno a la jerarquía cuando no existen posibilidades de resultar victorioso ${ }^{42}$. En situaciones de trauma o abandono, el valor evolucionista de la depresión reside en la posibilidad de terminar con un estado de angustia de separación prolongado y, por lo tanto, evitar los efectos psicobiológicos deletéreos ${ }^{43}$. En ese sentido se postula que la depresión corresponde a una respuesta ante la amenaza de los vínculos afectivos, lo que se traduce en una alteración de la capacidad de mentalización respecto a los deseos y motivaciones propias o de los demás $^{44,45}$. Específicamente, el apego inseguro se ha relacionado con una mayor vulnerabilidad para el desarrollo de depresión y conducta suicida ${ }^{46}$. Por lo tanto, existe una estrecha relación entre el estilo de apego, el estrés y la mentalización en la etiopatogenia de la depresión ${ }^{47}$.

En consecuencia, pensamos que el conocimiento de los mecanismos etiopatogénicos de la depresión es importante para el médico ya que le permite abordar su tratamiento contando con un modelo comprensivo que considere, por ejemplo, la relevancia de la carga genética, la necesidad de considerar el antecedente de trauma infantil o el valor adaptativo que puede tener el desánimo y la angustia.

\section{Personalidad y depresión}

La relación entre el temperamento como manifestación de la personalidad y las alteraciones del ánimo proviene de la antigüedad griega. En la actualidad, se ha desarrollado el concepto de temperamentos afectivos (depresivo, hipertímico, ciclotímico, irritable y ansioso) como manifestaciones subclínicas de algún trastorno del espectro afectivo $^{48}$. Dichos temperamentos han mostrado ser universales, con características distintivas y sin diferencias de género ${ }^{49}$. Tellenbach desarrolló el concepto de Typus melancholicus ${ }^{50}$ para referirse a un conjunto de rasgos del carácter que determinan la personalidad premórbida en la depresión melancólica. Más recientemente, Sidney Blatt ${ }^{51}$ ha elaborado un modelo comprensivo de la depresión cuyo principal supuesto es que la cualidad de la experiencia depresiva depende de la personalidad cuyo desarrollo ocurre en una interacción dialéctica y sinérgica entre la tendencia hacia la autodefinición y las relacionales interpersona$\operatorname{les}^{52}$. Estas dimensiones han sido denominadas respectivamente como introyectiva (autonomía/ perfeccionismo) y anaclítica (dependencia/sociotropía). Ambas dimensiones se asocian con distintas estructuras de personalidad, diferentes estilos relacionales y de apego, una vulnerabilidad ante eventos ambientales específicos (fracaso versus pérdida), una presentación clínica determinada y una respuesta característica ante los tratamientos farmacológicos o psicoterapéuticos ${ }^{53}$. Consecuentemente, la depresión es entendida como una forma de psicopatología que no deriva sólo de una alteración biológica inespecífica sino como un modo maladaptativo de tramitar las disrupciones severas y persistentes del desarrollo ${ }^{54}$ en el contexto de un trastorno interpersonal más que intrapersonal ${ }^{55}$.

Por lo anterior, el abordaje clínico del paciente deprimido deberá considerar siempre los aspectos situacionales y caracterológicos, en especial cuando nos encontremos frente a un paciente de difícil diagnóstico o manejo.

\section{Discusión}

Los trastornos psiquiátricos son cuadros etiológicamente complejos; por lo tanto, para entenderlos es necesario contar con un modelo que, sin dejar de lado la rigurosidad conceptual y empírica, sea pluralista e integrativo ${ }^{56}$ y cuyo fundamento se encuentre en la estrecha colaboración con otras ramas de la ciencia ${ }^{57}$.

El diagnóstico en psiquiatría supone la existencia de distintos puntos de vista o enfoques (positivista, fenomenológico o hermenéutico), que involucran, de parte del médico, diferentes niveles de compromiso cognitivo, afectivo y persona ${ }^{58}$ y que culmina en una negociación entre la subjetividad del paciente y la del terapeuta ${ }^{59}$. Sin embargo, sabemos que el diagnóstico depende también del contexto sociocultural y de la estructura de los sistemas de salud que funcionan en diálogo con las familias, los medios de masas y las administraciones locales ${ }^{60}$. Por lo tanto, uno de los desafíos para el desarrollo de nuevos sistemas nosológicos consiste en incorporar variables que permitan refinar los diagnósticos, integrando la evidencia neurocientífica con los hallazgos clínicos de manera de establecer grupos de trastornos con 
criterios compartidos -y, posiblemente, también con etiologías o vulnerabilidades comunes- en base al riesgo genético, asociación familiar, sustratos neurales, marcadores biológicos y endofenotipos ${ }^{61}$.

En la actualidad, el modelo médico de causalidad, focalizado en esencias biológicas simples, ha resultado insuficiente para captar la naturaleza de las enfermedades mentales; por el contrario, su adecuada conceptualización requiere de múltiples perspectivas explicativas que considere, además, la interacción de sus elementos en distintos niveles $^{62}$. Por lo mismo, a pesar de los indudables esfuerzos por desarrollar recomendaciones clínicas de calidad, consideramos que las guías utilizadas actualmente en nuestro país ${ }^{63}$ no incorporan en su totalidad las complejidades inherentes al diagnóstico de la depresión.

Dado que las enfermedades mentales no corresponden a entidades monocausadas, una clasificación ateórica y categorial, basada en conductas y síntomas observables que no considere la etiopatogenia es insuficiente para dar cuenta de la complejidad y riqueza del fenómeno clínico que llamamos depresión. Por el contrario, la nosología psiquiátrica debiera incorporar un enfoque que integre la observación clínica con las hipótesis causales $^{64}$.

La depresión es un trastorno complejo, etiológicamente multideterminado y clínicamente heterogéneo al cual se puede llegar a través de diversas vías, cuyas manifestaciones psicopatológicas se relacionan con la personalidad y que depende de la interacción entre factores genéticos y ambientales a lo largo del desarrollo ${ }^{65}$. Independiente del mecanismo etiopatogénico involucrado en su origen y de las diversas formas que puede tomar, la "verdadera" depresión mantiene una coherencia fenomenológica entre sus manifestaciones, el estilo de personalidad y el contexto sociocultural ${ }^{66}$. Siguiendo con lo anterior, consideramos que la depresión es un trastorno afectivo que se manifiesta clínicamente por un descenso del estado de ánimo, anhedonia, baja autoestima y un aumento de la autocrítica y los niveles de perfeccionismo. A este núcleo sintomático se puede llegar por diversas vías (ambientales, neurobiológicas, caracterológicas) con distintos grados de interacción ${ }^{67}$. Según la manera en que se relacionan estas vías la depresión puede tomar diversas formas: psicótica y melancólica (predominio factores biológicos), no melancólica (predominio de elementos caracterológicos), anaclítica/introyectiva (personalidad, configuración de estilos de apego), etc. Este modelo permite refinar los diagnósticos, planificar mejor los tratamientos y determinar el pronóstico.

En consecuencia, planteamos que el abordaje diagnóstico de la depresión consiste en un proceso secuencial (Tabla 1) que debe responder una serie de preguntas cuyo objetivo final será determinar por qué este individuo se encuentra deprimido en este preciso momento ${ }^{68}$. Llegamos entonces a la conclusión de que el diagnóstico de depresión deberá ser de exclusión, habiendo descartado previamente bipolaridad, enfermedades médicas, otros trastornos psiquiátricos como el consumo de sustancias y enfermedades comórbidas.

Las hipótesis en las que se basan las clasificaciones actuales serán mejoradas cuando se integre el conocimiento que la genética y las neuroimágenes están aportando a la comprensión no sólo de la etiopatogenia de las enfermedades mentales sino también de la forma en que actúan las intervenciones terapéuticas desde los fármacos a la psicoterapia ${ }^{69}$. En ese sentido, la búsqueda de marcadores biológicos específicos podrá ayudar no sólo a definir la presencia y severidad de los síntomas sino también a delimitar subtipos clínicos, predecir la respuesta al tratamiento y establecer un pronóstico. Las clasificaciones futuras debieran incorporar elementos que permitan distinguir perfiles clínicos y neurobiológicos específicos como ocurre, por ejemplo, con la depresión vinculada a eventos traumáticos en la infancia.

En muchos casos, especialmente cuando se trata de cuadros de difícil manejo, o que no responden adecuadamente a los tratamientos estandarizados en protocolos, los clínicos tienden a "desmedicalizar" la depresión incorporando otras variables en su aproximación diagnóstica como la personalidad, la cultura y el entorno social en un proceso dinámico de "negociación" que se realiza junto con el paciente. Por otra parte, según los supuestos de la medicina basada en evidencias, uno de los factores determinantes al momento de tomar decisiones clínicas no es sólo el conocimiento científico acerca de la patología sino también las preferencias del paciente ${ }^{70}$. Por lo tanto, considerando las múltiples dimensiones a través de las cuales es posible entender el fenómeno clínico que llamamos depresión -desde la genética y la neurobiología hasta la personalidad 


\section{Tabla 1. Recomendaciones para el abordaje diagnóstico de la depresión}

\begin{tabular}{|c|c|c|}
\hline Paso & Recomendación & Preguntas \\
\hline 1 & $\begin{array}{l}\text { Ante la sospecha de depresión, el médico debe explorar la } \\
\text { presencia de la tríada sintomática: desánimo, baja autoestima } \\
\text { y autorreproches. Luego, debe descartar causas orgánicas y } \\
\text { bipolaridad, ubicando dichos síntomas dentro del espectro } \\
\text { afectivo (bipolar-monopolar) a través del estudio de los ante- } \\
\text { cedentes familiares, la historia de hipomanía y el perfil clínico. } \\
\text { Finalmente, debe definir la presencia de subtipos clínicos: } \\
\text { depresión psicótica, melancólica o no melancólica }\end{array}$ & $\begin{array}{l}\text { ¿Tiene el paciente una depresión? } \\
\text { ¿Su depresión es de origen orgánico o psiquiátrico? } \\
\text { ¿Su depresión es de tipo monopolar o bipolar? } \\
\text { ¿A qué subtipo de depresión monopolar corres- } \\
\text { ponde? }\end{array}$ \\
\hline 2 & $\begin{array}{l}\text { Una vez que ha confirmado la existencia de depresión y la } \\
\text { ha ordenado de acuerdo con la recomendación anterior, el } \\
\text { médico debe considerar la manera en que la personalidad del } \\
\text { paciente influye en la presentación clínica (anaclítica/introyec- } \\
\text { tiva) y la eventual respuesta a los tratamientos. En este punto } \\
\text { debiera estudiarse el antecedente de trauma infantil }\end{array}$ & $\begin{array}{l}\text { ¿Qué tipo de organización de la personalidad pre- } \\
\text { domina en el paciente: anaclítica o introyectiva? } \\
\text { ¿De qué manera la personalidad ha determinado, } \\
\text { condicionado o perpetuado la sintomatología? }\end{array}$ \\
\hline 3 & $\begin{array}{l}\text { Explorar las situaciones desencadenantes y el contexto re- } \\
\text { lacional en que se desarrollan los síntomas (experiencias de } \\
\text { pérdida o fracaso) y el grado de reactividad que presentan }\end{array}$ & $\begin{array}{l}\text { ¿De qué naturaleza es la situación ante la cual el } \\
\text { paciente está reaccionando o "defendiéndose"? } \\
\text { ¿Qué costos ha tenido el problema para el pa- } \\
\text { ciente? } \\
\text { ¿Qué consecuencias para su vida pudiera tener un } \\
\text { cambio psicológico o emocional? }\end{array}$ \\
\hline 4 & $\begin{array}{l}\text { Considerar el entorno social, cultural y su relación con los } \\
\text { sistemas de salud involucrados }\end{array}$ & $\begin{array}{l}\text { ¿De qué manera el ambiente, la cultura, el entorno } \\
\text { social y los sistemas de salud en que se encuentra el } \\
\text { paciente influyen sobre la aparición de los síntomas, } \\
\text { su persistencia o la respuesta a las intervenciones? }\end{array}$ \\
\hline 5 & $\begin{array}{l}\text { Establecer un diagnóstico y una indicación diferencial de los } \\
\text { tratamientos que involucre un proceso de "negociación" } \\
\text { entre las propias teorías etiopatogénicas del paciente, sus } \\
\text { expectativas de tratamiento y el juicio del clínico acerca de la } \\
\text { naturaleza del problema }\end{array}$ & $\begin{array}{l}\text { ¿Cuál será la "hipótesis de trabajo" que guiará la } \\
\text { toma de decisiones entre el paciente y el terapeuta? } \\
\text { ¿Qué tratamiento es el más indicado para este } \\
\text { paciente en particular? } \\
\text { ¿Qué resultados se espera obtener con el trata- } \\
\text { miento? }\end{array}$ \\
\hline 6 & $\begin{array}{l}\text { Por último, el médico debe considerar la depresión como } \\
\text { un trastorno al cual se puede llegar desde diversas vías rela- } \\
\text { cionadas entre sí (biología, personalidad, ambiente, etc.) y } \\
\text { que, según la manera en que se den estas combinaciones, se } \\
\text { presenta clínicamente a través de distintos subtipos (psicóti- } \\
\text { ca, melancólica, no melancólica; anaclítica/introyectiva) cuya } \\
\text { diferenciación tiene implicancias diagnósticas, pronósticas y } \\
\text { terapéuticas }\end{array}$ & $\begin{array}{l}\text { ¿De qué manera este paciente en particular llegó } \\
\text { a deprimirse? }\end{array}$ \\
\hline
\end{tabular}

y la conducta, incorporando las variables ambientales, sociales y culturales- el proceso diagnóstico consistirá en determinar lo que, para cada paciente en particular (con sus propios requerimientos, fortalezas y vulnerabilidades), significa padecer una enfermedad depresiva. Sólo así podremos implementar tratamientos que, fundamentados en una indicación diferencial (ya se trate de fármacos o psicoterapia) resulten ser cada día más efectivos.

\section{Referencias}

1. Angst J. A regular review of the long term follow up of depression. BMJ 1997; 315: 1143-6.

2. Andrade L, Caraveo-Anduaga JJ, Berglund P, Bijl RV, De Graaf R, Vollebergh W, et al. The epidemiology of major depressive episodes: results from the International Consortium of Psychiatric Epidemiology (ICPE) Surveys. Int J Methods Psychiatr Res 2003; 12: 3-21. 
3. World Health Organization. The Global Burden of Disease: 2004 Update (WHO, 2008).

4. Alarcón R. Culture, cultural factors and psychiatric diagnosis: review and projections. World Psychiatry 2009; 8: 131-9.

5. Rush AJ. Problems associated with the diagnosis of depression. J Clin Psychiatry 1990; (51): Suppl: 15-22.

6. Cepoiu M, McCusker J, Cole MG, Sewitch M, Belzile E, Ciampi A. Recognition of depresión by non-pychiatricphysicians: a systematic literature review and metaanalysis. J Gen Int Med 2008; 23: 25-6.

7. Katon W, Simon G, Russo J, Von Korff M, Lin EH, Ludman E, et al. Quality of depression care in a populationbased sample of patients with diabetes and major depression. Med Care 2004; 42: 1222-9.

8. Kohn R, Saxena Sh, Levav I, Saraceno B. The treatment gap in mental healthcare. Bull World Health Organ 2004; 82: 858-66.

9. Kendler KS. Introduction: why does psychiatry need philosophy? In: Kendler KS, Parnas J (eds): Philosophical Issues in Psychiatry. Explanation, Phenomenology, and Nosology. Baltimore, Johns Hopkins University Press, 2008, p. 1-16.

10. Berríos G, Porter R. A history of clinical psychiatry. London, The Athlone Press, 1995.

11. Berríos G. Historia de los síntomas de los trastornos mentales. La psicopatología descriptiva desde el siglo XIX. México, D.F., Fondo de Cultura Económica, 2008.

12. Desseilles M, Chang T, Piguet C, Bertschy G, Dayer A. A Three-dimensional model of thoughts: insight into depression. Psychopathology 2012; 45: 203-14.

13. Goldberg D. The heterogeneity of "major depression". World Psychiatry 2011; 10 (3): 226-8.

14. Leuchter A, Cook I, Hunter A, Korb A. A new paradigm for the prediction of antidepressant treatment response. Dialogues Clinneurosci 2009; 11: 435-46.

15. Parker G. Beyond major depression. Psychol Med 2005; (35): 467-74.

16. Akiskal H, McKinney W. Depressive disorders: toward a unified hypothesis: clinical, experimental, genetic, biochemical, and neurophysiological data are integrated. Science 1973; 182: 20-9.

17. Parker G. Classifying Depression: Should Paradigms Lost Be Regained? Am J Psychiatry 2000; 157: 1195-203.

18. Parker G. Defining melancholia: the primacy of psychomotor disturbance. Acta Psychiatr Scand 2007; 115: 21-30.

19. Parker G. Crawford J. A spectrum model for depressive conditions: Extrapolation of the atypical depression prototype. J Affect Disord 2007; 103: 155-63.
20. Ghaemi N, Vöhringer P, Vergne D. The varieties of depressive experience: Diagnosing mood disorders. Psychiatr Clin N Am 2012; 35: 73-86.

21. Angst J, Marneros A. Bipolarity from ancient to modern times: conception, birth and rebirth. J Affect Disord 2001; 67: 3-19.

22. Angst J. The bipolar spectrum. Br J Psychiatry 2007; 190: 189-91.

23. Akiskal A, Pinto O. The evolving bipolar spectrum: Prototypes I, II, III and IV. Psychiatr Clin N Am 1999; 22: 517-34.

24. Ghaemi N, Ko Y, Goodwin F. "Cade's Disease" and Beyond: Misdiagnosis, Antidepressant Use, and a Proposed Definition for Bipolar Spectrum Disorder. Can J Psychiatry 2002; 47: 125-34.

25. Hirschfeld R. The Comorbidity of Major Depression and Anxiety Disorders: Recognition and Management in Primare Care. Prim Care Companion J Clin Psychiatry 2001; 3: 244-54.

26. Thakker J, Ward T. Culture and classification: the crosscultural application of the DSM-IV. Clin Psychol Rev 1998; 18 (5): 501-29.

27. Karasz A. Cultural differences in conceptual models of depression. Soc Sci Med 2005; (60): 1625-35.

28. Kleinman A. Culture and Depression. N Engl J Med 2004; 351 (10): 951-3.

29. Simon G, VonKorff M, Piccinelli M, Fullerton C, Ormel J. An international study of the relation between somatic symptoms and depression. N Engl J Med 1999; 341 : 1329-35.

30. Mann J. Clinical pleomorphism of major depression as a challenge to the study of its pathophysiology. World Psychiatry 2010; 9 (3): 167-8.

31. Sullivan P, Neale M, Kendler K. Genetic Epidemiology of Major Depression: Review and Meta-Analysis. Am J Psychiatry 2000; 157: 1552-62.

32. Hong C, Tsai T. The Genomic Approachesto Major Depression. Current Pharmacogenomics 2003; 1: 67-74.

33. Caspi A, Sugden K, Moffitt T, Taylor A, Craig I, Harrington $\mathrm{H}$, et al. Influence of Life Stress on Depression: Moderation by a Polymorphism in the 5-HTT Gene. Science 2003; 301: 386-9.

34. Austin M-P, Mitchell PH, Goodwin G. Cognitive deficits in depression: Possible implications for functional neuropathology. Br J Psychiatry 2001; 178: 200-6.

35. Heim Ch, Nemeroff Ch. The Role of Childhood Trauma in the Neurobiology of Mood and Anxiety Disorders: Preclinical and Clinical Studies. Biol Psychiatry 2001; 49: 1023-39.

36. Risch N, Herrell R, Lehner T, Liang K, Eaves L, Hoh J, et al. Interaction Between the Serotonin Transporter Gene 
(5-HTTLPR), Stressful Life Events, and Risk of Depression: A Meta-analysis. JAMA 2009; 301 (23): 2462-71.

37. Heim Ch, Plotsky $P$, Nemeroff Ch. Importance of studying the contributions of early adverse experience to neurobiological findings in depression. Neuropsychopharmacology 2004; 29: 641-8.

38. Krishnadas R, Cavanagh J. Depression: an inflammatory illness? J Neurol Neurosurg Psychiatry 2012; 83: 495502.

39. Freud S. Duelo y Melancolía. En: Obras completas. Buenos Aires: Editorial Amorrortu; 2000. p. 235-56.

40. Beck A. The evolution of the cognitive model of depression and its neurobiological correlates. Am J Psychiatry 2008; 165: 969-77.

41. Nettle D. Evolutionary origins of depression: a review and reformulation. J Affect Disord 2004; 81: 91-102.

42. Nesse R. Is depression an adaptation? Arch Gen Psychiatry 2000; 57: 14-20.

43. Watt D, Panksepp J. Depression: An evolutionary conserved mechanism to terminate separation distress? A review of aminergic, peptidergic, and neural network perspectives. Neuropsychoanalysis 2009; 11 (1): 7-51.

44. Allen J, Fonagy P, Bateman A. Mentalizing in clinical practice. Washington D.C.: American Psychiatric Publishing, Inc.; 1 edition; 2008.

45. Luyten P, Fonagy P, Lemma A, Target M. Depression. En: Bateman A, Fonagy P, ed. Handbook of mentalizing in mental health practice. American Psychiatric Association, Washington D.C.; 2012. p. 385-417.

46. Grunebaum M, Galfalvy H, Mortenson L, Burke A, Oquendo M, Mann J. Attachment and social adjustment: relationships to suicide attempt and major depressive episode in a prospective study. J Affect Disord 2010; 123 (1-3): 123-30.

47. Heim Ch, Newport J, Mletzko T, Miller A, Nemeroff Ch. The link between childhood trauma and depression: Insights from HPA axis studies in humans. Psychoneuroendocrinology 2008; 33: 693-710.

48. Akiskal K, Akiskal H, The theoretical underpinnings of affective temperaments: implications for evolutionary foundations of bipolar disorder and human nature. J Affect Disord 2005; 85: 231-9.

49. Vázquez G, Tondo L, Mazzarini L, Gonda X. Affective temperaments in general population: A review and combined analysis from national studies. J Affect Disord 2012; 139: 18-22.

50. Tellenbach H. Melancholie. Problemgeschichte, Endogenität, Typologie, Pathogenese, Klinik. Berlin: Springer; 1961.

51. Blatt S. Polarities of experience. Relatedness and selfdefinition in personality development, psychopathology, and the therapeutic process. Washington D.C.: American Psychological Association; 2008.

52. Blatt S, Luyten P. A structural-developmental psychodynamic approach to psychopathology: Two polarities of experience across the lifespam. Dev Psychopathol 2009; 21: 793-814.

53. Luyten P. Blatt S, Corveleyn J. Introduction. En: The theory and treatment of depression. Towards a dynamic interactionism model. Leuven University Press, Leuven, 2005. p. 5-15.

54. Luyten P, Blatt S, Corveleyn J. The convergence among psychodynamic and cognitive-behavorial theories of depression: Theoretical overview. En: The theory and treatment of depression. Towards a dynamic interactionism model. Leuven: Leuven University Press; 2005. p. 67-94.

55. Hammen C. Stress and depression. Annu Rev Clin Psychol 2005; 1: 293-319.

56. Kendler K. Toward a Philosophical Structure for Psychiatry. Am J Psychiatry 2005; 162: 433-40.

57. Musalek M, Larach-Walters V, Lépine J-P, Millet B, Gaebel W. On Behalf of The WFSBP Task Force on Nosology and Psychopathology. Psychopathology in the 21st century. World J Biol Psychiatry 2010; 11: 844-51.

58. Fuchs Th. Subjectivity and intersubjectivity in psychiatric diagnosis. Psychopathology 2010; 43: 268-74.

59. Stanghellini G. A hermeneutic framework for psychopathology. Psychopathology 2010; 43: 319-6.

60. Kirmayer L. Cultural variations in the clinical presentation of depression and anxiety: implications for diagnosis and treatment. J Clin Psychiatry 2001; 62: 22-8.

61. Kupfer D. Regier D. Neuroscience, clinical evidence, and the future of psychiatric classification in DSM-5. Am J Psichiatry 2011; 168 (7): 672-4.

62. Kendler K. Levels of explanation in psychiatric and substance use disorders: implications for the development of an etiologically based nosology. Mol Psychiatry 2012; 17 : 11-21.

63. Ministerio de Salud. Guía Clínica Depresión en personas de 15 años y más. Santiago, MINSAL, 2013.

64. Luyten P, Blatt S. Looking Back Towards the Future: Is It Time to Change the DSM Approach to Psychiatric Disorders? The Case of Depression. Psychiatry 2007; 70 (2): 85-99.

65. Luyten P, Blatt S, Corveleyn: Towards integration in the theory and treatment of depression? The time is now. En: The theory and treatment of depression. Towards a dynamic interactionism model. Leuven: Leuven University Press; 2005. p. 253-84.

66. Parnas J. A sea of distress. En: Kendler K, Parnas J (ed.). Philosophical issues in psychiatry II: Nosology. Oxford: 
La depresión como un diagnóstico complejo - A. Botto et al

Oxford University Press; 2012. p. 229-33.

67. Bleichmar H. El modelo modular-transformacional y los subtipos de depresión. En: Avances en psicoterapia psicoanalítica. Hacia una técnica de intervenciones específicas. Buenos Aires: Paidós; 2005.

68. Parker G. Manicavasagar V. Modelling and managing the depressive disorders. A clinical guide. Cambridge: Cambridge University Press; 2005. p. 14-20.
69. Buchheim A, Viviani R, Kessler H, Kächele H, Cierpka M, Roth G, et al. Changes in Prefrontal-Limbic Function in Major Depression after 15 Months of LongTerm Psychotherapy. PLoS ONE 2012; 7 (3): e33745. doi:10.1371/journal.pone.0033745

70. Sackett D, Rosenberg W, Gray J, Haynes R, Richardson W. Evidence based medicine: what it is and what it isn't. BMJ 1996; 312: 71-2. 